\section{Identifying pattern in global developmental delay children: A retrospective study at King Fahad specialist hospital, Dammam (Saudi Arabia)}

\author{
Hafiz Habibullah, Raidah Albradie, \\ Shahid Bashir \\ Pediatric Neurology, Neuroscience \\ Center, King Fahad Specialist Hospital \\ Dammam, Dammam, Saudi Arabia
}

\begin{abstract}
Global developmental delay (GDD) and intellectual disability are relatively common in pediatric neurology conditions. A retrospective study was designed to analyze risk factors and clinical features in children with GDD at our hospital. No previous data is available on GDD from Saudi Arabia. This study was conducted at king Fahad specialist hospital Dammam (KFSHD) of 134 GDD children (82, 61\% males, 52, 39\% females), (age 1-9 years). They were assessed by using Griffith Mental Development Scales for (0-2) years and 3-8 years old in locomotors, personal/social, communication, eye \& hand co-ordination, performance and practical reasoning. Patients with ASD and non-cooperative behavior were excluded. $75 \%$ had developmental delay since birth while $84 \%$ had no problem during pregnancy. $22 \%$ had birth weight below $2.5 \mathrm{~kg}$. $56 \%$ had epilepsy and $57 \%$ had interfamily marriages. $51 \%$ were diagnosed cases in the present study. $40 \%$ had genetic cause, $25 \%$ had metabolic problem, $58 \%$ had neuroradiology abnormality and $45 \%$ had EEG abnormalities. There a variety of delays in development (speech and language variant, global delay, and the motor variant) noted and are commonly seen in a clinical practice in KFSHD. Longitudinal research beginning in early development will help to understand the developmental domains and neurological comorbidities in these children at high risk for neurodevelopmental disorders.
\end{abstract}

\section{Introduction}

Global developmental delay (GDD) is term used for children under 5 years of age. It is defined as a significant delay in two or more domains of development, including activities of daily living as well as motor, cognitive, speech/language, and personal/social skills. ${ }^{1,2}$ The clear diagnosis of GDD is problematic because of its heterogeneous etiology; thus, the causes of GDD are undetermined in approximately $62 \%$ of children. ${ }^{3}$ The exact prevalence of GDD is not known precisely, however from different studies it is estimated 1-3\%.1,3,4 It is probably higher in Saudi Arabia due to high rate of consanguinity. Individuals in whom genetic causes are established generally undergo very exhaustive, expensive, and often invasive diagnostic evaluations, even though the results may not change the medical or therapeutic management of the delay.

Significant controversy still surrounds the symptom complex of GDD in term of diagnosed in children under the age of 5 years. Since formal psychometric testing often cannot be reliably undertaken in the young child a clear-cut, objective threshold for diagnosis, as in the case of cognitive delay, often cannot be clearly established.4,5 Therefore, GDD diagnosis relies on the summation of clinical findings in several developmental areas. Another cause for controversy is the apparent wide variability of clinical presentations that fall under the GDD diagnostic umbrella. Term Learning difficulty/disability is used for children older than 5 years of age. Mild learning difficulty (IQ 50-70) affects $1-2 \%$ of the child population. Severe learning difficulty (IQ < 50 ) affects $0.3-0.5 \%$ and may be subdivided into moderate (IQ 35-49), severe (IQ 20-34) and profound learning difficulty (IQ $<20) .6$

Although on established guidelines, studies were conducted in North America, Europe and Turkey.7,8 Not much data is available about the GDD in the Gulf or local populations. This study was conducted to determine the etiologic yield for GDD in children attending a tertiary care hospital in this part of the world. To address these questions, we evaluated clinic referred subject in KFSHD by correlating detail clinical history, examination, developmental assessment, behavioral assessment, and results of relevant investigations in a developmental clinic.

\section{Materials and Methods}

A detailed retrospective chart review of 134 children diagnosed with GDD in pediatric neurology and development assessment clinic at King Fahd specialist hospital, Dammam (KFSH-D) between 1 Feb 2016 and 1 April 2018 was undertaken (i.e. 1-9 years). Permission was obtained from the local ethic committee of KFSHD. Children were identified by review of the charts of all patients assessed in the Developmental Progress Clinic during the time period stud-
Correspondence: Shahid Bashir, King Fahad Specialist Hospital, Ammar Bin Thabit St, Al Muraikabat, Al Dammam 32253, Saudi Arabia. E-mail: sbashir10@gmail.com

Key words: Speech and language, fine motor, neurodevelopmental disorder.

Acknowledgements: The authors extend their appreciation to participants take part in the study.

Contributions: HH, SB analyses the data and they were involved in drafting the manuscript. $\mathrm{HH}$ and RA contributed substantially to the experiment design. HH, RA and SB participated in drafting and writing the manuscript. MS. All authors read and approved the final manuscript.

Conflict of interest: The authors declare no potential conflict of interest.

Funding: None.

Received for publication: 21 July 2019.

Revision received: 9 September 2019.

Accepted for publication: 9 September 2019.

This work is licensed under a Creative Commons Attribution NonCommercial 4.0 License (CC BY-NC 4.0).

CC Copyright: the Author(s), 2019

Licensee PAGEPress, Italy

Pediatric Reports 2019; 11:8251

doi:10.4081/pr.2019.8251

ied. Referrals to the clinic derive mainly from pediatricians, family physicians and pediatric neurology in the eastern province region. The population seen reflects a wide range of local ethnic and socio-economic backgrounds. All children referred to the clinic undergo a formal multidisciplinary developmental assessment (complete history, and developmental pediatrics examination). A diagnostic conclusion from these evaluations is drawn after the first or second visit to the clinic.

A diagnosis of GDD was ascribed to all children presenting with significant delays (defined as performance is below the mean on age-appropriate, standardized, norm-referenced testing) in two or more developmental domains. As part of standard clinical protocol, all children found to have GDD undergo a formal cognitive assessment with a trained pediatric physician or clinical psychologist.

\section{Assessment scale}

These children were developmentally assessed by using Griffith mental development scales for 0-8 years old in Locomotor, personal/social, Hearing and language, eye 
and hand co-ordination, performance and practical reasoning scales.

Patients with autism and non-cooperative were excluded.

A clinical sheet was designed by the research group to get detailed history, particularly birth, developmental, family, and relevant past medical including epilepsy and medication history.

A considerable part of sheet was assigned for clinical examination particular neurological examination and development assessment in locomotor, personal/social, hearing and language, eye $\&$ hand co-ordination, performance and practical reasoning scales. Severity of development delay in each domain was labelled as mild, moderate and severe categories according to the developmental age assessed by developmental pediatrician using Griffith scale compared with actual chronological age (i.e.; mild: 67-100\%; moderate: 33-66\%; and severe $<33 \%$ ).

The results of investigations like genetic, metabolic, neuro-imaging and EEG were collected from medical records.

\section{Inclusion and exclusion criteria}

Inclusion criteria: children of 1-9 years of age presenting with development delay.

Exclusion criteria: patients with autism and non-cooperative for development assessment. Children whose development level was above 8 years of age as Griffith scale measures development age only up to 8 years. Absence of the complete data set of the patient as designed in the clinical evaluation sheet.

\section{Statistical analysis}

Developmental scores were recorded as centiles for the following variables fine motor performance, expressive vocabulary, and receptive language. All data gathered were analyzed using SPSS software version 22.0 (SPSS Inc., Chicago). A P-value of 0.05 or less was considered to be statistically significant.

\section{Results}

A total of 134 children initially diagnosed with GDD were identified over the study inclusion criteria. The age range of children was between 1-9 years (Figure 1). Of these, 134 children (82 males, 52 females, Figure 2). Among all $89 \%$ had developmental delay/learning difficulties including $11 \%$ had behavioral problems.

The data derived from their mother pregnancies were normal $(n=113,84 \%)$, while rest $(n=21,16 \%)$ showed $(n=6,4.7 \%)$ bleeding, $(n=3,2.2 \%)$ decreased fatal movements, $(n=3,2.2 \%)$ polyhydromnios, $(n=3,2.2 \%)$ diabetes mellitus, $(n=3,2.2 \%)$ hypertension/pre-eclampsia and $(\mathrm{n}=2$, $1.4 \%$ ) epilepsy problems. During the labor $(n=128,96 \%)$ had spontaneous onset delivery. The data showed $(n=112,84 \%)$ normal vaginal delivery, $(n=20,15 \%)$ had caesarean section, while $(n=2,1 \%)$ had ventouse delivery.

These kids $(n=30,22 \%)$ had birth weight below $2.5 \mathrm{~kg},(\mathrm{n}=18,13 \%)$ needed resuscitation and $(n=14,10 \%)$ were intubated for few days. During the neonatal period, 11 patients had neonatal seizures, 6 had sep- sis, 18 had severe jaundice and 14 of them needed phototherapy. Only $(n=20,15 \%)$ children had feeding difficulties and needed

Table 1. Underlying etiology in global developmental delay.

\begin{tabular}{lll} 
Cases with abnormalities & No. & $\%$ \\
Metabolic & 34 & 25 \\
Genetic & 55 & 41 \\
\hline Neuroradiology & 76 & 57 \\
EEG & 60 & 45 \\
\hline
\end{tabular}

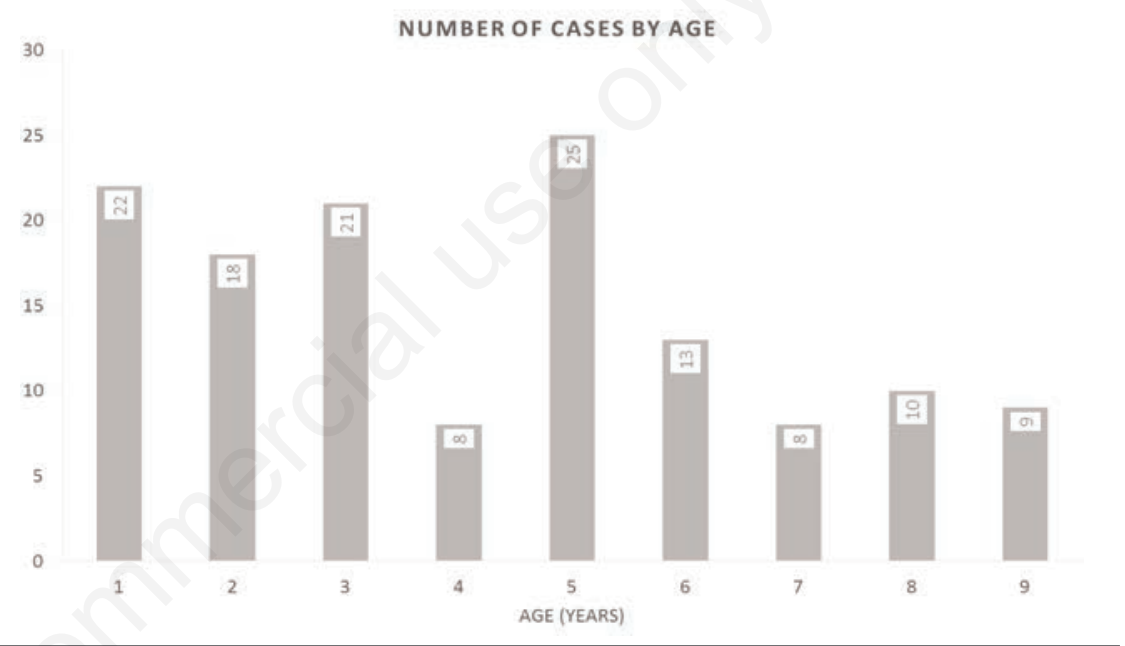

Figure 1. Number of cases by age.

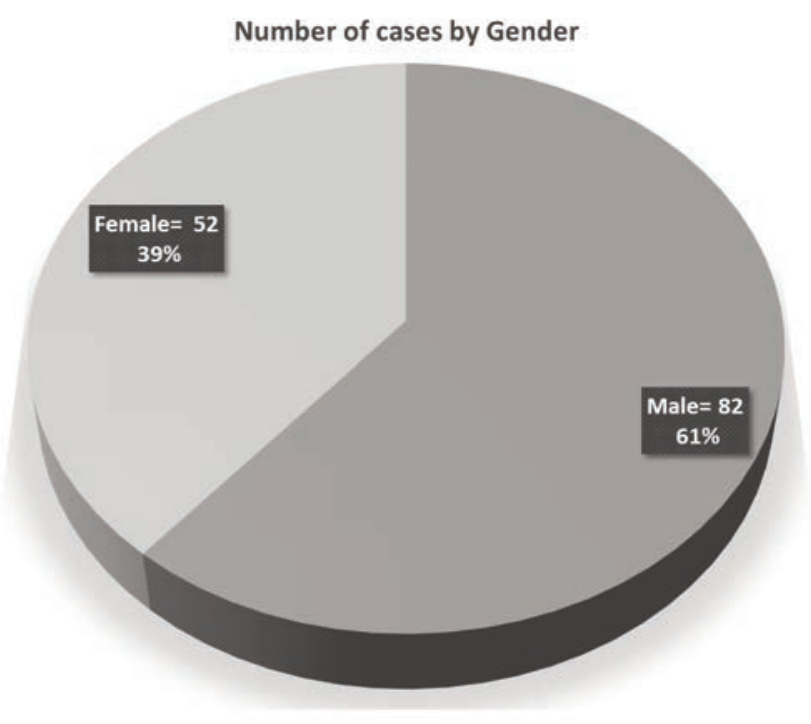

Figure 2. Number of cases by gender group. 
feeding assistance. Family history showed $57 \%$ had consanguinity (Figure 3 ). 25\% had epilepsy in close relatives, $16 \%$ had learning disability in family, $20 \%$ had relatives went to special school, $7 \%$ had mental health problems in the family and $6 \%$ had hearing difficulties in family. GDD kids' group $(56 \%)$ showed various types of epilepsy (Figure 4).

\section{Developmental assessment}

The developmental assessment showed GDD particularly in speech and locomotors, while $21 \%$ had development regression and $79 \%$ had development delay since birth.

Overall severe development delay was $47 \%$, moderate delay $18 \%$, mild delay $16 \%$ and normal $6 \%$ in this group data. In locomotor assessment task- severe development delay was $(n=37,28 \%$, moderate delay $(n=22,16 \%)$, mild delay $(n=24,18 \%)$ and $(\mathrm{n}=11,8 \%)$ showed normal function of locomotor in study group (Figure 5).

For the personal/social assessment, the severe development delay was in $(\mathrm{n}=32)$, moderate delay $(n=27)$ mild delay $(n=21)$ and normal $(n=12)$ in this study group (Figure 5). For the hearing and language assessment, $(n=33)$ were severely delayed, $(n=25)$ moderately delayed, $(n=21)$ mild delay and normal $(n=7)$ in task.

The data showed $(n=32)$ were severely delayed, $(n=23)$ had moderate delay, $(n=23)$ had mild delay and normal $(n=8)$ in Eye and hand coordination assessments (Figure 5).

Performance task showed $(\mathrm{n}=30)$ had severe developmental delay, $(n=24)$ had moderate delay, $(n=20)$ were mildly delayed and $(n=8)$ were with in normal range during performance task (Figure 5).

Growth parameters showed 23\% had head circumference below 3 rd. centile. While $42 \%$ of our patients had some form of dysmorphic features. $15 \%$ of our patients showed neurocutaneous abnormalities.

\section{Diagnosis}

Sixty-nine $(51 \%)$ out of 134 were clinically diagnosed by neurologist (Figure 6). Genetic disease being the most common $41 \%$, Metabolic abnormalities were found in $25 \%$, Neuro radiological abnormalities were found in $57 \%$ and EEG abnormalities were found in $45 \%$ cases (Table 1 ).

\section{Discussion}

This study was hospital based and does not represent the community-based study. There is possibility of bias towards working up of more affected children. The $51 \%$ children with GDD, which was within the range

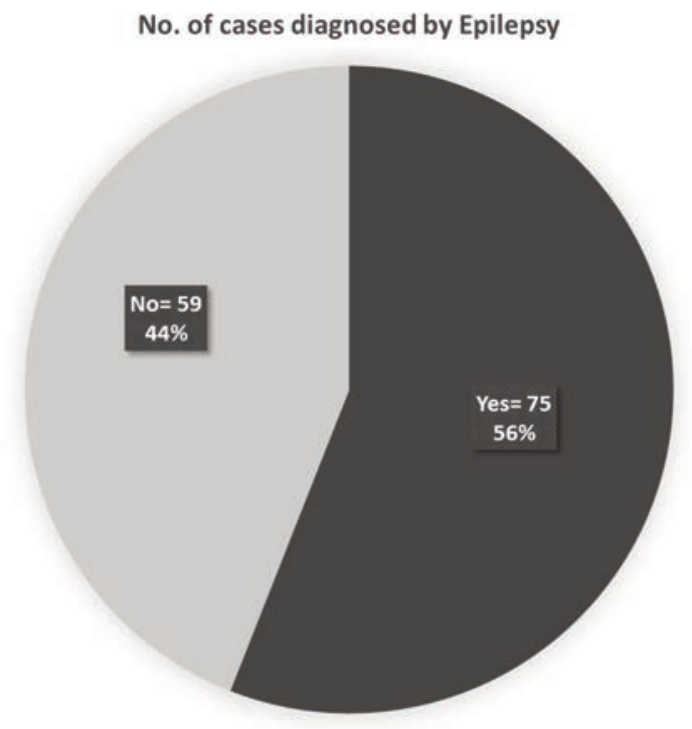

Figure 3. Number of cases by consanguinity.

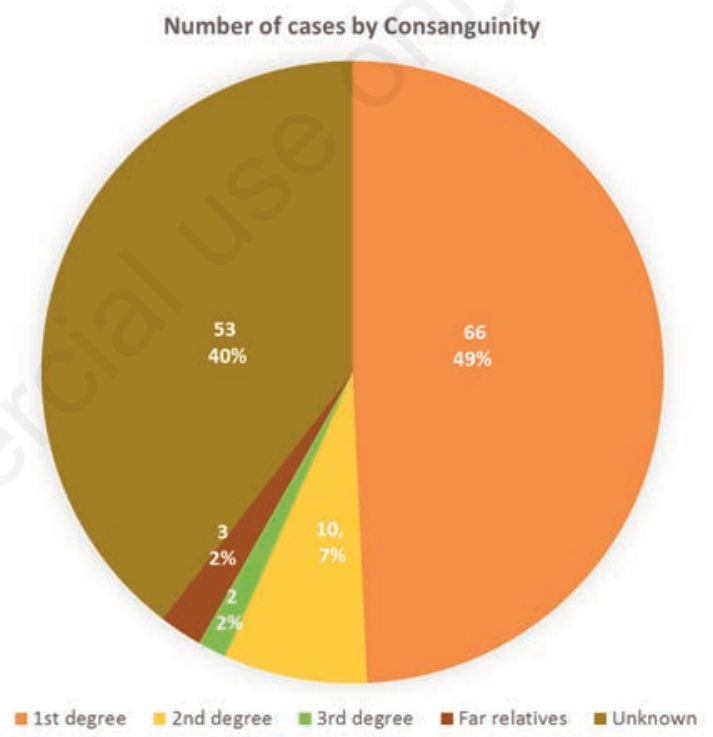

Figure 4. Number of cases diagnosis by epilepsy.

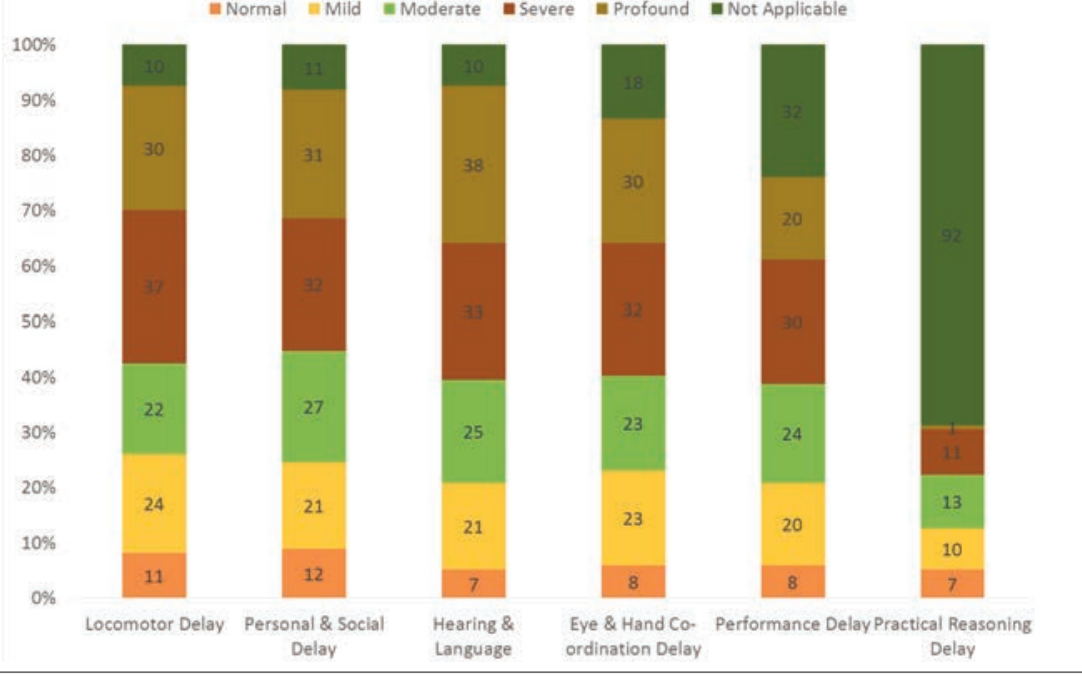

Figure 5. Overall development trajectory in development delay group. 
reported from previous studies. 7,8 Three previous studies from a single center in North America had reported a yield of $63 \%$, $55 \%$, and $38 \% .{ }^{9}$ In a retrospective study from Turkey on 247 patients with GDD, etiology was found in $63 \% .10$ In a study from Jordan, the rate was identified in $44.5 \%$ only. ${ }^{11}$ These differences in the yield are explained by the differences in the population, criteria used, and the extent of investigations. Another factor which has a major impact on detection of the underlying cause is the high incidence of consanguineous marriages resulting in familial cases in this part of the world. ${ }^{11}$ Few studies showed half of the children with delays, especially language- related delays, are not detected before starting school and do not use a standardized instrument for neurodevelopmental assessment.12,13 Recent guidelines strongly recommend the systematic screening of developmental delay in young children, in order to provide early beneficial interventions to the child and family. $7-10$

The diagnostic work-up with regard to prenatal history, perinatal data, and clinical examination was complete in 94 to $99 \%$, and laboratory investigations in 80 to $93 \%$ of the 134 children ascertained for analysis, Perinatal asphyxia, Genetic, metabolic diseases and cerebral digenesis were the common causes of GDD, while familial consanguinity, dimorphism and non-specific white matter changes in MRI guided more than $50 \%$ of the etiology of GDD. This distribution was similar to reports from previous studies. ${ }^{7-9}$

Developmental delay is a symptom not a diagnosis. It is very important to listening to parents, paying special attention to their concerns, and doing a meticulous history makes the greatest contribution to a neurodevelopmental diagnosis. ${ }^{12,14}$

It is clear that establishing a diagnosis enables us to answer questions on: why it has happened (etiology), what does it mean for our child (prognosis), what treatments might be available (precision medicine) and whether it can be prevented in the future (prenatal testing and preimplantation genetic diagnosis). Major malformations for syndrome identification should be obvious to the clinician on physical examination. ${ }^{15-17}$ Careful neurodevelopmental examination should include observations of minor neurological signs, associated movements, fine motor skills, and gait.

A diagnosis guides the clinician in looking for rare but treatable diseases and helps predict the risk of a similar condition occurring in future pregnancies. ${ }^{16-18}$ Helping a family organize a plan to address children developmental delay is essential. Directing the child to early intervention services is a sound first start. Any child less than 3 years of age who has a physical or mental condition that has a high probability of resulting in developmental delay is eligible for services, so a neurodevelopmental diagnosis helps qualify a child for early intervention services. ${ }^{19,20}$ Services are mandated for infants, toddlers and preschoolers. Although eligibility criteria vary by jurisdiction, common measures of delay are $25 \%$ and/or two standard deviations in one or more of the developmental domains. ${ }^{20}$ Recognizing developmental patterns that guide a neurodevelopmental assessment is essential to help families get the support services they need. ${ }^{20}$

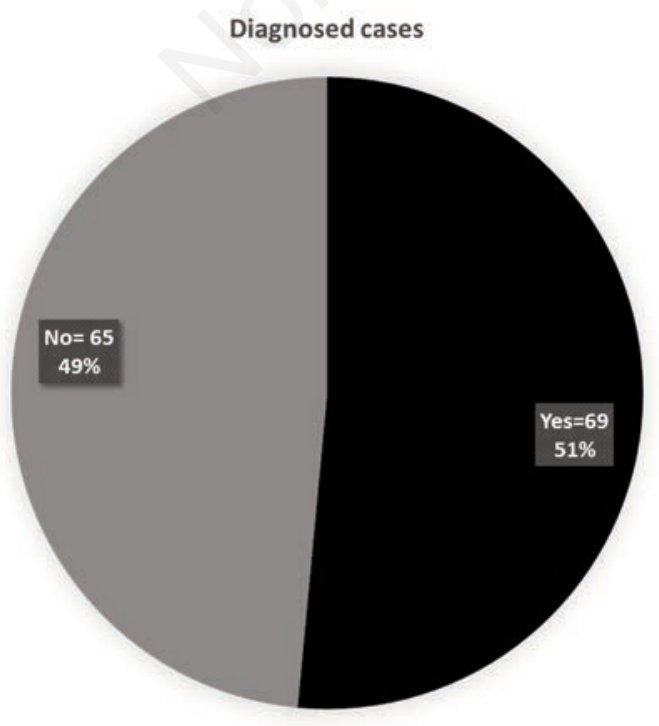

Figure 6. Number of diagnosis cases.

It is worth mentioning that psychosocial deprivation, toxin intake, head trauma, and child abuse, shaken baby syndrome resulting in developmental delay were looked into during this study; however, none were found, which may the study is conducted over a short period. 20

One limitation of the study is its retrospective design. However, we believe that the overall structure of the local Developmental Progress Clinic, the clinical source of all our study participants, limits potential biases, as all patients undergo the same standardized evaluations, recorded in a predetermined uniform format.

\section{Conclusions}

The study gives a baseline data about the spectrum of GDD in the region. In addition, specific screening investigations should be obtained on the basis of clinical suspicion in order to improve the yield. All patients with developmental delay/learning disability need a comprehensive medical evaluation by complete medical history with particular detail of birth, early development and family history. Physical examination with particular emphasis on growth parameters, dysmorphism, neurocutaneous stigmata, systemic and neurological examination. Development assessment by using standardized development assessment scales. If the clinical diagnosis is obvious or suspected, confirm the diagnosis with appropriate genetic testing. If diagnosis is unknown and no clinical diagnosis is strongly suspected, begin in stepwise evaluation by: chromosomal microarray; Fragile $\mathrm{X}$ testing; for females complete $M E C P 2$ gene study; metabolic testing; brain MRI if clinically indicated; EEG if epilepsy.

Whether diagnosed or not, results and their implications should be carefully explained to the parents/care givers. Appropriate support and referral to appropriate support services should be made.

\section{References}

1. Shevell M, Majnemer A, Platt RW, et al. Developmental and functional outcomes in children with global developmental delay or developmental language impairment. Dev Med Child Neurol 2005;47:678-83.

2. Shevell M, Ashwal S, Donley D, et al. Practice parameter: evaluation of the child with global developmental delay: report of the Quality Standards Subcommittee of the American 
Academy of Neurology and The Practice Committee of the Child Neurology Society. Neurology 2003;60:367-80.

3. Jimenez-Gomez A, Standridge SM. A refined approach to evaluating global developmental delay for the international medical community. Pediatr Neurol 2014;51:198-206.

4. American Association on Mental Retardation. Mental retardation: definition, classification and systems of supports 10th edn. Washington, DC: American Association on Mental Retardation; 2002.

5. Johnson JH, Goldman J. Developmental assessment in clinical child psychology. New York, NY: Pergamon Press, 1990.

6. Whiting K. Investigating the child with learning difficulty. Curr Paediatr 2001;11:240-7.

7. Majnemer A, Shevell MI. Diagnostic yield of the neurologic assessment of the developmentally delayed child. J Pediatr 1995;127:193-9.

8. Battaglia A, Bianchini E, Carey JC. Diagnostic yield of the comprehensive assessment of developmental delay/mental retardation in an institute of child neuropsychiatry. Am J Med Genet 1999;82:60-6.
9. Shevell MI, Majnemer A, Rosenbaum P, Abrahamowicz M. Etiologic determination of childhood developmental delay. Brain Dev 2001;23:228-35.

10. Ozmen M, Tatli B, Aydinli N, et al. Etiologic evaluation in 247 children with global developmental delay at Istanbul, Turkey. J Trop Pediatr 2005;51:310-3.

11. Palfrey J, Singer JD, Walker DK, Butler JA. Early identification of children's needs: a study of five metropolitan communities. J Pediatr 1987;111:651-9.

12. Masri A, Hammamy H, Khreisat A. Profile of developmental delay in children under five years of age in a highly consanguineous community: A hospital-based study - Jordan. Brain Dev 2010.

13. Bear LM. Early identification of infants at risk for developmental disabilities. Pediatr Clin North Am 2004;51:685701.

14. Rydz D, Shevell MI, Majnemer A, Oskoui M. Developmental screening. J Child Neurol 2005;20:4-21.

15. Capute AJ, Accardo PJ. A neurodevelopmental perspective on the continuum of developmental disabilities. In: Capote AJ, Accardo PJ, eds. Developmental Disabilities in Infancy and Childhood. 2nd ed. Baltimore: Paul H.
Brookes; 1996. pp 1-22.

16. Polifka JE, Friedman JM. Medical genetics: 1. Clinical teratology in an age of genomics. CMAJ 2002;167:265-73.

17. Hunter AG. Medical genetics: 2. The diagnostic approach to the child with dysmorphic signs. Can Med Assoc J 2002;167:367-72.

18. Committee on Genetics. Molecular genetic testing in pediatric practice: a subject review. Pediatrics 2000;106: 1494-7.

19. Shevell M, Ashwal S, Donley D, et al. Practice Parameter: Evaluation of the Child with Global Developmental Delay: Report of the Quality Standards Subcommittee of the American Academy of Neurology and The Practice Committee of the Child Neurology Society, Quality Standards Subcommittee of the American Academy of Neurology, Practice Committee of the Child Neurology Society. Neurology 2003;60:367-80.

20. Committee on Children with Disabilities. The pediatrician's role in the development and implementation of an individual education plan (IEP) and/or an individual family service plan (IFSP). Pediatrics 1999;104:124-7. 